\title{
Symmetry Analysis of Options Pricing with Transactions Costs Driven by Fractional Brownian Noises
}

\author{
Nteumagn BF, Pindza E and Mare $E^{*}$ \\ Department of Mathematics and Applied Mathematics, University of Pretoria, Pretoria 0002, Republic of South Africa
}

\begin{abstract}
We provide a closed-form solution for the European and Asian option pricing models when the source of randomness is a fractional Brownian motion as opposed to the geometric Brownian motion. In addition to the source of randomness, transaction costs are considered to be non-negligible. For the case of the European option, proportional transaction costs hide in the volatility and do not change the form of the model. The construction of the solution is based on the symmetries of the model. The model for Asian options has an additional parameter that makes the volatility time-dependent, which complicates the solution process. However, we are still able to obtain solutions using Lie symmetry methods.
\end{abstract}

Keywords: Lie symmetries; European option; Fractional brownian; Transaction cost

\section{Introduction}

In the construction of several models, we make explicit and implicit assumptions. However, quite often, when we solve these models, we overlook these assumptions or they are simply "diluted" in the process [1]. For models encoded as differential equations, assumptions that translate into the invariance of underlying equations are particularly useful in the search of their solutions. This is the main thrust of Lie symmetry analysis of differential equations pioneered by Sophus Lie [2-18]. In such analysis, one algorithmically looks for infinitesimal transformations (i.e., transformations depending on a small parameter and enjoying additional properties $[2,9,17]$ of both dependent and independent variables that does not change the underlying differential equation. These transformations are then systematically used for finding solutions.

An infinitesimal transformation of both dependent and independent variables that leaves a differential equation unchanged up to the first-order in the small parameter is called an infinitesimal symmetry [17] of this equation. For a given differential equation, the set of all its infinitesimal symmetries form an infinitesimal group (Lie algebra in the modern terminology) [19]. Perhaps the most useful property of a symmetry of a differential equation is that it transforms a solution into another one. Thus, for instance, by starting with a trivial solution, one may construct a non-trivial one by acting on the former with a symmetry [2]. Also, for equations belonging to the same family, those that can be invertibly mapped to each other have isomorphic symmetry algebra. We shall employ this last property for transforming a complicated equation into one which is easier to solve [20]. Lie symmetry analysis has a broad range of applications. This includes among others, mathematical biology, mathematical physics and financial mathematics to name just a few. In this paper, we focus our attention on the application of Lie symmetry analysis to financial mathematics, and more precisely to the theory of option pricing.

Option pricing and replication has attracted both the business and the academic audience since the development of the Nobel price winning Black-Scholes differential equation. Significant success has been registered in the attempt to solve the model numerically. However, it is not always possible to find practical numerical algorithms to solve higher dimensional differential equations. Because of its ability to reduce the dimension of the problem, Lie symmetries analysis promises to be a very good instrument to mitigate Bellman's "curse of dimensionality" [21]. It constitutes a great tool for analytical approximation of the solution even when numerical analysis has not been found to be conclusive. Thus far, it has been applied to the celebrated Black-Scholes equation with success.

$$
\frac{\partial u}{\partial t}+\frac{\partial u}{\partial x}+\frac{\sigma^{2}}{2} x^{2} \frac{\partial^{2} u}{\partial x^{2}}-r u=0
$$

Indeed, Gazizov and Ibragimov [22] paved the way in the application of Lie symmetry analysis to solve financial models [23] Their research was based on the basic BlackScholes equation (1.1). In their work, they applied Lie symmetries to transform the BlackScholes equation into the heat equation and solved it. Pooe et al. [24] deduced a fundamental solution to zero-coupon bonds. Since the introduction of Lie symmetries to finance, many models have been designed to price and hedge options accurately, and models are becoming more sophisticated with the development of new techniques and the evolvement of technology (see for example, $[12,13]$ ).

The option pricing theory from inception rested on the arbitrage argument which stipulates that by continuously hedging a portfolio of stocks and risk-free bonds, one can exactly replicate the return on a stock [25]. By doing so, the option value must then be equal to that of the replicating portfolio $[5,23,11]$. In reality, one cannot completely hedge a portfolio of stocks and bonds without incurring any costs. This is because continuous trading and replication has been proven to be very expensive, even though it is possible to consider very small proportional transaction cost [11,26-28]. The consideration of transaction costs in option pricing is more recent as assumptions of the classical models have minimized these costs in the past. The derivation

${ }^{*}$ Corresponding author: Eben Mare, Department of Mathematics and Applied Mathematics, University of Pretoria, Pretoria 0002, Republic of South Africa, Tel: +27-12-420-4111; E-mail: eben.mare@up.ac.za

Received July 18, 2017; Accepted July 25, 2017; Published July 31, 2017

Citation: Nteumagn BF, Pindza E, Mare E (2017) Symmetry Analysis of Options Pricing with Transactions Costs Driven by Fractional Brownian Noises. J Appl Computat Math 6: 356. doi: 10.4172/2168-9679.1000356

Copyright: (C) 2017 Nteumagn BF, et al. This is an open-access article distributed under the terms of the Creative Commons Attribution License, which permits unrestricted use, distribution, and reproduction in any medium, provided the original author and source are credited. 
of the option pricing equation relies heavily on the random process followed by the underlying stock price. In the case of the celebrated Black-Scholes equation, one assumes that the stock follows the classical geometric Brownian motion [5,24]. This process assumes independent increments, which makes it different from the fractional Brownian motion $(\mathrm{fBm})$ where there is a serial correlation in the increments. This correlation allows for predictability in the model. The similarity between the two processes is the fact that they are both geometric.

It has been shown [8] that random changes can be accurately measured by the use of a parameter known as the Hurst parameter. The construction of a dam on the Nile river in the early 1950s saw the birth of the Hurst exponent. The measure of the water levels of increase or decrease was known to follow a random walk. In other words, the next level of increase or decrease is independent of the previous one. However, Edwin Hurst, hydrologist in the Nile dam project, showed that the long term memory of the system indicates an autocorrelation of the time series of water levels, and the rate at which these decrease as the lag between pairs of values increase [8]. The movement of stock price is similar to this model. We therefore exploit the results by Leland [11] for the derivation of our model. Earlier in 2014, we achieved an accurate numerical solution of our model with exponential convergence ([19]) by applying spectral methods to analyze the problem. In this paper, we use Lie symmetries to determine the exact solution, which sets us a step closer to the calibration of our model.

We first give some preliminaries on Lie symmetries of differential equations in Section 2. Secondly, we determine the symmetries of the European and Asian option pricing models and obtain their analytical solutions in Section 3. In Section 4 we apply the Lie symmetry technique to reduce the PDEs and provide solutions. Finally, we provide concluding remarks in Section 5.

\section{Lie Symmetries and Equivalence Transformations}

Our aim in this section is to provide a brief introduction to Lie symmetry algorithm and define what are equivalence transformations. We shall limit ourselves to scalar second-order PDEs as our models will fall in this category. We shall sometimes sacrifice mathematical rigor for the sake of clarity.

\section{Lie symmetry algorithm}

Classical methods for solving differential equations are ad-hoc in nature. Lie symmetry analysis offers a unified approach to the solution of differential equations and the reduction of the number of dependent variables. Intuitively, a symmetry of a differential equation is an invertible change of independent and dependent variables that leave the underlying equation unchanged. Finding all the symmetries of a given differential equation is a difficult if not impossible task in many situations. However, as Lie [2,17] discovered, those symmetries that depend on a small parameter and which form a local one-parameter group [2,17] can be systematically calculated in fairly general situations. In this subsection, we specialize Lie's symmetry algorithm to the class of problems that interest us in this work. Also, we highlight a few properties of Lie symmetries. Consider a second-order partial differential equation (PDE) in one dependent variable $u$ and two independent variables $\mathrm{t}$ and $\mathrm{x}$.

$$
\mathrm{E}\left(\mathrm{t}, \mathrm{x}, \mathrm{u}, \mathrm{u}_{(1)}, \mathrm{u}_{(2)}\right)=0
$$

where $E$ is a sufficiently smooth function of its argument, $u_{(1)}=\{u t, u x\}$, $\mathrm{u}_{(2)}=\{\mathrm{utt}, \mathrm{utx}, \mathrm{uxx}\}$, and the indexes stands for partial differentiations. An invertible transformation of both the independent and dependent variables

$$
\begin{aligned}
& \bar{t}=t+\varepsilon T(\mathrm{t}, \mathrm{x}, \mathrm{u})+\mathrm{O}\left(\varepsilon^{2}\right), \\
& \overline{\mathrm{x}}=\mathrm{x}+\varepsilon \xi(\mathrm{t}, \mathrm{x}, \mathrm{u})+O\left(\varepsilon^{2}\right), \\
& \bar{u}=u+\varepsilon \eta(\mathrm{t}, \mathrm{x}, \mathrm{u})+\mathrm{O}\left(\varepsilon^{2}\right)
\end{aligned}
$$

is an infinitesimal symmetry or Lie symmetry if

$$
\mathrm{E}\left(\mathrm{t}, \mathrm{x}, \mathrm{u}, \mathrm{u}_{(1)}, \mathrm{u}_{(2)}\right)=\mathrm{O}\left(\in^{2}\right) \text {. }
$$

Whenever, eqn. (2.2) is satisfied, $\bar{u}_{(1)}=\left\{\bar{u}_{\overline{\mathrm{t}}}, \bar{u}_{\bar{x}}\right\}$, and $\bar{u}_{(2)}=\left\{\bar{u}_{\bar{t}}, \bar{u}_{\overline{t x}}, \bar{u}_{\overline{x x}}\right\}$

In order to obtain a practical definition of infinitesimal symmetry, we need to transform eqn. (2.6) into an equivalent form by means of Taylor's expansion in $\epsilon$. Thus, we must obtain first the Taylor expansions of the derivatives appearing in eqn. (2.6). This is our immediate goal.

We may compute the differential $\mathrm{d}^{-} \mathrm{u}$ in two different ways. The first way comes from the very definition of a differential:

$$
\begin{aligned}
& d \bar{u}=\bar{u}_{t} \bar{d} t+\bar{u}_{x}, d_{\bar{x}}, \\
& =\left[\bar{u}_{i}\left(1+\varepsilon D_{t} \tau\right)+\varepsilon \bar{u}_{x} D_{t} \xi\right] d t+\left[\varepsilon D_{x} \tau \bar{u}_{i}+\bar{u}_{x}\left(1+\varepsilon \mathrm{D}_{x} \xi\right)\right] \mathrm{dx}+\mathrm{O}\left(\varepsilon^{2}\right) .
\end{aligned}
$$

Where we have used eqns. (2.3)-(2.4), Dt and Dx are the operators of total differentiations with respect to $t$ and $x$ respectively:

$$
\begin{aligned}
& D_{t}=\frac{\partial}{\partial \theta}+u_{t} \frac{\partial}{\partial y_{\theta}}+u_{t t} \frac{\partial}{\partial u_{\partial}}+u t_{x} \frac{\partial}{\partial u_{\partial}}+\ldots \ldots . ., \\
& D_{x}=\frac{\partial}{\partial x}+u_{x} \frac{\partial}{\partial u}+u_{x t} \frac{\partial}{\partial u_{t}}+u_{x x} \frac{\partial u_{x}}{\partial u}+\ldots . .,
\end{aligned}
$$

The second way of computing $\mathrm{d}^{-} \mathrm{u}$ is by using eqn. (2.5):

$$
d u=\left(\mathrm{u}_{t}+\varepsilon \mathrm{D}_{t} \eta\right) \mathrm{dt}+\left(\mathrm{u}_{x}+\varepsilon \mathrm{D}_{x} \eta\right) \mathrm{dx}+\mathrm{O}\left(\varepsilon^{2}\right)
$$

From eqn. (2.7) and eqn. (2.10), we infer the following system of two linear equations in the two unknowns $\overline{\mathrm{U}}_{\mathrm{t}}$ and $\overline{\mathrm{U}}_{\mathrm{x}}$

$$
\begin{aligned}
& \left(1+\varepsilon \mathrm{D}_{t} \tau\right) \overline{\mathrm{u}}_{\bar{t}}+\varepsilon \bar{u}_{x} D_{t} \xi=u_{t}+\varepsilon D_{t} \eta, \\
& \in D_{x} \tau \bar{u}_{\bar{t}}+\left(1+\in D_{x} \xi\right)=u_{x}+\in D_{x} \eta
\end{aligned}
$$

By solving the system in eqn. (2.11)-(2.12), we obtain

$$
\bar{u}_{\bar{t}}=u_{t}+\in \eta^{u t}+O\left(\epsilon^{2}\right) \text {, }
$$$$
\bar{u}_{\bar{x}}=u_{x}+\in \eta^{u x}+O\left(\epsilon^{2}\right) \text {, }
$$

where

$$
\begin{aligned}
& \eta^{u t}=D_{t}(\eta)-u_{t} D(\tau)-u_{x} D_{t}(\xi), \\
& \eta^{u x}=D_{x}(\eta)-u_{x} D_{t}(\tau)-u_{x} D_{t}(\xi),
\end{aligned}
$$

By replacing ${ }^{-} \mathrm{u}$ with ${ }^{-} \mathrm{u}^{-} \mathrm{t}$ or ${ }^{-} \mathrm{u}^{-} \mathrm{x}$ in the preceding calculations, we arrive at the following expansions of the second derivatives:

$$
\begin{aligned}
& \bar{u}_{t \bar{t}}=u_{t t}+\in \eta^{u t t}+O\left(\epsilon^{2}\right) \\
& \bar{u}_{t \bar{x}}=u_{t x}+\in \eta^{u t t}+O\left(\epsilon^{2}\right) \\
& \bar{u}_{x \bar{x}}=u_{x x}+\in \eta^{u t t}+O\left(\epsilon^{2}\right)
\end{aligned}
$$

in which $\eta^{u_{t u}}, \eta^{u_{x}}$ and $\eta^{u_{x x}}$ are given by

$$
\begin{aligned}
& \eta^{u_{t t}}=D_{t}\left(\eta^{u_{t}}\right)-\mathrm{u}_{t t} D_{t}(\tau)-\mathrm{u}_{t x} D_{t}(\xi) \\
& \eta^{u_{t x}}=D_{t}\left(\eta^{u_{x}}\right)-\mathrm{u}_{t x} D_{t}(\tau)-\mathrm{u}_{x x} D_{t}(\xi) \\
& =D_{t}\left(\eta^{u_{t}}\right)-\mathrm{u}_{t t} D_{x}(\tau)-\mathrm{u}_{t x} D_{x}(\xi) \\
& \eta^{u_{x x}}=D_{x}\left(\eta^{u_{x}}\right)-\mathrm{u}_{t x} D_{t}(\tau)-\mathrm{u}_{x x} D_{t}(\xi)
\end{aligned}
$$

The equations (2.15)-(2.16) are known as first-order prolongation formulas whereas Equation. (2.20)-(2.22) are the second-order prolongation formulas. Note the pattern between first order and 
second-order prolongation formulas. Such insight may be utilized to infer high order prolongation expressions.

Now let us expand eqn. (2.6) to the second-order in $\epsilon$ :

$$
E\left(\mathrm{t}, \mathrm{x}, \mathrm{u}, \mathrm{u}_{(1)}, \mathrm{u}_{(2)}\right)+\in X^{[2]}(\mathrm{E})=\mathrm{O}\left(\in^{2}\right)
$$

whenever eqn. (2.2) is satisfied, where

$$
\begin{aligned}
& X=\tau(\mathrm{t}, \mathrm{x}, \mathrm{u}) \frac{\partial}{\partial t}+\xi(\mathrm{t}, \mathrm{x}, \mathrm{u}) \frac{\partial}{\partial x}+\eta(\mathrm{t}, \mathrm{x}, \mathrm{u}) \frac{\partial}{\partial u} \\
& X^{[1]}=X+\eta^{u t} \frac{\partial}{\partial u_{t}}+\eta^{u x} \frac{\partial}{\partial u_{x}} \\
& X^{[2]}=X^{[1]}+\eta^{u_{t}} \frac{\partial}{\partial u_{t t}}+\eta^{u_{t x}} \frac{\partial}{\partial u_{t x}}+\eta^{u_{x x}} \frac{\partial}{\partial u_{x x}}
\end{aligned}
$$

We shall also refer to $\mathrm{X}$ as infinitesimal symmetry of eqn. (2.2). Now, eqn. (2.23) implies that

$$
X^{[2]}(\mathrm{E})=0
$$

Whenever eqn. (2.2) is satisfied. Equation (2.27) together with the phrase "whenever eqn. (2.2) is satisfied" may be written compactly as

$$
X^{[2]}(\mathrm{E})_{E q .(4.2)}=0
$$

Conversely, it can be shown (see for instance $[2,3,17]$ ) that if eqn. (2.2) can be locally solved with respect to one of its highest derivative and eqn. (2.28) is satisfied, so is eqn. (2.6). Therefore, modulo the aforementioned local solvability condition, eqn. (2.28) characterizes infinitesimal symmetries of eqn. (2.2). That is why it is called the linearized symmetry condition for infinitesimal symmetries.

After substituting one of the highest derivatives of eqn. (2.2) into the linearized symmetry condition (2.28), we end up with an equation which is a polynomial in the remaining derivatives of $u$. The latter equation splits into an over-determined system of linear PDEs for the unknowns $\tau, \xi$ and $\eta$. There are several computer algebra packages for generating automatically this set of linear PDES $[12,13]$ so that one seldom generates them by hand.

Owing the structure of the determining equation (2.28), the infinitesimal symmetries enjoy the following additional properties:

- If $X_{1}$ and $X_{2}$ are two Lie symmetries of eqn. (2.2) and $c_{1}$ and $c_{2}$ are real numbers, then $c_{1} X_{1}+c_{2} X_{2}$ is also Lie symmetries of (2.2).

- If $X_{1}$ and $X_{2}$ are two Lie symmetries of eqn.(2.2), then their Lie bracket

$$
\left[\mathrm{X}_{1}, \mathrm{X}_{2}\right]=\mathrm{X}_{1}\left(\mathrm{X}_{2}\right)-\mathrm{X}_{2}\left(\mathrm{X}_{1}\right)
$$

is also a Lie symmetry of eqn. (2.2).

Thanks to the above two properties, the collection of all the infinitesimal symmetries of eqn. (2.2) form a Lie algebra [2,16,17].

\section{Equation depending on arbitrary parameters: equivalence transformations}

Here we focus on introducing equivalence transformations and we emphasize some of their applications to problems pertinent to this paper. As, in the previous subsection, we shall limit ourselves to a scenario relevant to this work. Notations that are not introduced here are those of the previous sections. For an in-depth study of the equivalence problem, we refer the reader to the work of Ovsiannikov [18] and former students [2,17]. Consider a family of differential equations
$\mathrm{E}\left(\mathrm{t}, \mathrm{x}, \mathrm{u}, \mathrm{u}_{(1)}, \mathrm{u}_{(2)}, \Lambda\right)=0$,

which is parameterized by a set $\Lambda$ made possibly of arbitrary constants and arbitrary functions of $\mathrm{t}, \mathrm{x}, \mathrm{u}$ and $\mathrm{u}_{(1)}$. An equivalence transformation of eqn. (2.29) is an invertible change of dependent and independent variables which preserves its differential structure. The key difference between a symmetry and an equivalence transformation is that, an equivalence transformation is required only to preserve the structure of the set of arbitrary parameters $\Lambda$ : If $\Lambda$ contains an arbitrary number $\alpha$, then any equivalent equation's parameter set, $\bar{\wedge}$ , must contain an arbitrary parameter ${ }^{-} \alpha$ at the same position in the differential structure of the equation. If $\Lambda$ contains a function $\mathrm{f}(\mathrm{t}, \mathrm{x}, \mathrm{u})$, then the parameter set of the equivalent equation, $\bar{\wedge}$, must contain a function $\bar{f}(\overline{\mathrm{t}}, \overline{\mathrm{x}}, \overline{\mathrm{u}})$ at the same location in the differential structure of the equation. It is straightforward to see that a symmetry of eqn.(2.29) is always and equivalence transformation of eqn. (2.29). However, the converse is not in general true. Also, two equations of the class in eqn. (2.29) that can be mapped to each other via an equivalence transformation have isomorphic Lie symmetry algebras [17,2,16,3]. The latter results are often used to detect isomorphic equations within the class in eqn. (2.29) and to even construct explicitly the equivalence transformation which realizes this isomorphic.

Equivalence transformations induce an equivalence relation on the family of equations (2.29): Two equations of the family are equivalent if and only if they can be mapped to each other by using an equivalence transformation. Finding all the equivalence classes modulo this equivalence transformation is the group classification problem associated to eqn. (2.29). In the group classification problem, one seeks very simple representative of each equivalence class, which in general, carries fundamental properties of its members. It is generally good to choose a simple enough representative to permit subsequent analysis. Indeed, once the properties of a representative are elucidated, they can always be mapped to those of a given representative via an appropriate equivalence transformation. This is mainly how we are going to exploit equivalence transformations in this work.

The above narrative begs two questions of practical importance: How does one find all the equivalence transformations of the family (2.29)? How does one verify that two equations of the family in eqn. (2.29) are equivalent?

The problem of calculating all the equivalence transformations of the family in eqn. (2.29) is as difficult as finding all its symmetries. In some particular instance, one may find all the equivalence transformations through the use of the chain rule and reasoning about the differential structure of the family $[2,3,16,17]$. As for Lie symmetries, if we restrict ourselves to continuous groups of equivalence transformations, one arrives at an algorithm [16] which is similar to Lie's symmetry algorithm in its derivation and application. Concerning the issue of the equivalence of two equations of the family in eqn. (2.29), one uses the fact that two equation are equivalence if and only if their symmetry Lie algebras are isomorphic i.e. they have the same structure constants after an appropriate choice of their bases $[2,3,16,17]$. We shall take advantage of this result in the sequel.

\section{Mathematical Modeling of Vanilla European and Asian Options Driven by Fractional Brownian Motions}

This section is dedicated to the derivation of both vanilla European and Asian options PDEs under fractional Brownian noises. We only provide a detailed derivation of the former since the latter follows the same logic mutatis mutandis. 
Citation: Nteumagn BF, Pindza E, Mare E (2017) Symmetry Analysis of Options Pricing with Transactions Costs Driven by Fractional Brownian Noises. J Appl Computat Math 6: 356. doi: 10.4172/2168-9679.1000356

Page 4 of 8

\section{Black-Scholes model under fractional Brownian motion}

We provide the derivation of the European option pricing PDE in fractional Brownian motion with transactions costs.

Definition: A fractional Brownian motion $(\mathrm{fBm}) \mathrm{BH}(\mathrm{t})$ with Hurst exponent $H \in(0,1)$ is a Gaussian process with mean zero and covariance [4].

$$
E\left(\mathrm{~B}_{t}^{H}, \mathrm{~B}_{x}^{H}\right)=\frac{1}{2}\left(x^{2 H}+t^{2 H}-|t-x|^{2 H}\right)
$$

Under this model, the trader predicts a price for the stock at time $t$ as

$S(\mathrm{t})=\mathrm{S}_{0} \exp \left(\mu t+\sigma B^{H}(\mathrm{t})-\frac{\sigma^{2}}{2} t^{2 H}\right)$

which implies a stock model

$$
d S=\mu S d t+\sigma d B^{H}(\mathrm{t})
$$

We assume that the European call V $(\mathrm{t}, \mathrm{S})$ on the underlying stock $\mathrm{S}$ at time $\mathrm{t}$ with exercise $\mathrm{T}$ and exercise price $\mathrm{E}$ (also known as the strike price or just strike) has boundary conditions.

$$
V(\mathrm{~T}, \mathrm{~S})=\max \{\mathrm{S}(\mathrm{T})-E, 0\}, \mathrm{V}(\mathrm{t}, 0)=0, \lim _{s \rightarrow+\infty} V(\mathrm{t}, \mathrm{s})=\mathrm{S} .
$$

Indeed, if at maturity, the stock price is greater than the strike price, then the option is exercised. If instead the stock price is less than the strike, then the buyer exercises his right not to buy and the option is worthless.

We also considers the risk free bond price $\mathrm{D}(\mathrm{t})$ whose evolution is given by

$$
\mathrm{dD}(\mathrm{t})=\mathrm{rD}(\mathrm{t}) \mathrm{dt}
$$

We build a simple portfolio with value $\Pi$, say, made of stocks and risk-free bonds. We suppose that it costs us a proportion $\mathrm{k}$ of the turnover to replicate our portfolio (see for example [11]). If we replicate the portfolio with $\mathrm{X}_{1}(\mathrm{t})$ units of stocks and $\mathrm{X}_{2}(\mathrm{t})$ units of risk free bonds, then the portfolio $\Pi$ will be valued at

$$
\Pi=\mathrm{X}_{1}(\mathrm{t}) \mathrm{S}+\mathrm{X}_{2}(\mathrm{t}) \mathrm{D}(\mathrm{t})
$$

Assume small changes $\delta \Pi, \delta \mathrm{S}$ and $\delta \mathrm{D}$ in $\Pi, \mathrm{S}$ and $\mathrm{D}$, respectively, caused by a small change in time $\delta$ t. This change in the portfolio value is obtained at a transaction cost, which is assumed to be proportional to turnover, i.e., $\frac{k}{2}\left|\delta X_{1}(\mathrm{t})\right| S$. Hence we have from (3.35), we have

$$
\delta \Pi=X_{1}(\mathrm{t}) \delta \mathrm{S}+X_{2}(\mathrm{t}) \delta \mathrm{D}(\mathrm{t}) \mathrm{B}^{H}(\mathrm{t})-\frac{k}{2}\left|\delta X_{1}(\mathrm{t})\right| S
$$

Where $\delta \mathrm{D}(\mathrm{t})$ is a small change in risk-free bonds, $\delta \mathrm{X} 1(\mathrm{t})$ a small change in the number of stocks held. The replication interval $\delta t$ is chosen to be minimal. Therefore, by the Taylor theorem [26], expanding V in the $\delta$ - neighbourhood of $\mathrm{t}$ we can write

$$
\begin{aligned}
& \begin{aligned}
\delta D= & r D(\mathrm{t}) \delta t+O(\delta t)^{2} \\
\delta V & =\left(\frac{\partial V}{\partial t}+\mu S \frac{\partial V}{\partial S}\right) \delta t+\sigma S \frac{\partial V}{\partial S} \delta B^{H}(\mathrm{t})+\frac{\sigma^{2}}{2} S^{2} \frac{\partial^{2} V}{\partial S^{2}}\left(\mathrm{~B}^{H}(\mathrm{t})\right)^{2} \\
& +\frac{\sigma^{2}}{2} S^{2} \frac{\partial V}{\partial S}\left(\mathrm{~B}^{H}(\mathrm{t})\right)^{2}+O\left((\delta \mathrm{t})^{2 H}\right)
\end{aligned} \\
& \text { and } \\
& \begin{aligned}
\delta X_{1}(\mathrm{t}, \mathrm{s})=\frac{\partial X_{1}(\mathrm{t})}{\partial t} \delta t+\frac{\partial X_{1}(\mathrm{t})}{\partial S} \delta S+\frac{S^{2} \partial^{2}}{2} \frac{\partial^{2} X_{1}(\mathrm{t})}{\partial S^{2}}(\delta S)^{2} \\
\quad+\frac{1}{2} \frac{\partial^{2} X_{1}(\mathrm{t})}{\partial t^{2}}(\delta \mathrm{t})^{2}+\frac{\partial^{2} X_{1}(\mathrm{t})}{\partial t \partial S}(\delta \mathrm{t})(\delta S)+O\left((\delta \mathrm{t})^{3}\right)
\end{aligned}
\end{aligned}
$$

Now we have

$$
\delta S=S \sigma B^{H}(\mathrm{t})+\mu \mathrm{S} \delta \mathrm{t} .
$$

so

$$
\begin{aligned}
& E\left|(\delta S)^{2}\right|=S^{2}\left(\mathrm{e}^{\sigma\left(\frac{1}{2} \delta \mathrm{B}^{H}(\mathrm{t})\right)^{2}+\mu \delta t}-1\right) \\
& =S^{2}\left(\sigma^{2}(\delta t)^{2 H}+\mathrm{O}\left((\delta t)^{2 H}\right)\right)
\end{aligned}
$$

Similarly,

$$
\begin{aligned}
& E\left|(\delta S)^{2}\right|=S^{2}\left(\mathrm{e}^{\sigma\left(\frac{1}{2} \delta B^{H}(\mathrm{t})\right)^{2}+\mu \delta t}-1\right)^{2} \\
& =S^{2}\left(\sigma^{2}(\delta \mathrm{t})^{2 H}+\mathrm{O}\left((\delta \mathrm{t})^{2 H}\right)\right)
\end{aligned}
$$

$E\left|(\delta S)^{2}\right|=\mathrm{O}\left((\delta t)^{2 H}\right)$ for the higher even powers of $\delta \mathrm{S}$, and since $(\delta \mathrm{t})$ is chosen to be very small, higher powers are negligible.

Equation (3.38) gives

$$
\left|\delta X_{1}(\mathrm{t}, \mathrm{S})\right|=\sigma S\left|\frac{\partial X_{1}(\mathrm{t})}{\partial S}\right| \delta B^{H}(\mathrm{t}) \mid+O(\delta t)
$$

Similarly, (3.37) and (3.36) yield

$$
\delta \Pi=r X_{2}(\mathrm{t}) \mathrm{D}(\mathrm{t}) \delta \mathrm{t}+\mathrm{X}_{1}(\mathrm{t}) \delta S-\sigma \frac{k}{2}\left|\frac{\partial X_{1}(\mathrm{t})}{\partial S}\right|\left|\delta B^{H}(\mathrm{t})\right|+O(\delta t)
$$

If $\mathrm{V}(\mathrm{t}, \mathrm{S})$ is the value of the option replicated by the above portfolio $\Pi$, i.e., $V=\Pi$ to reduce arbitrage and remain consistent with economic equilibrium [26,27], then by WickIto's lemmas as in ref. [7]

$$
\delta V=\frac{\partial V}{\partial S} \delta S+\left(\frac{\partial V}{\partial t}+\frac{\sigma^{2}}{2} \mathrm{~S}^{2} \frac{\partial^{2} V}{\partial S^{2}}\right) \delta t
$$

Since $\mathrm{V}=\Pi, \delta \mathrm{V}=\delta \Pi$. From (3.35) and (3.36), we have

$$
\begin{aligned}
& \Delta=\frac{\partial V}{\partial S} \\
& r B^{H}(\mathrm{t}) \delta t-\frac{k}{2}|\delta \Delta| S=\left(\frac{\partial V}{\partial t}+\frac{\sigma^{2}}{2} \frac{\partial^{2} V}{\partial S^{2}}\right) \delta t
\end{aligned}
$$

So we have $\mathrm{V}=\Delta \mathrm{S}+\mathrm{B}^{\mathrm{H}}(\mathrm{t})$ and thus

$$
B^{H}(\mathrm{t})=V-\frac{\partial V}{\partial S} S
$$

Hence, by the fractional Leland [11] approximation

$$
\frac{k}{2}|\delta \Delta| S \approx \frac{\sigma^{2}}{2} \operatorname{Le}(\mathrm{H}) \mathrm{S}^{2}\left|\frac{\partial^{2} V}{\partial S^{2}}\right| \delta t
$$

Where $L e(\mathrm{H})=\sqrt{\frac{2}{\pi}}\left(\frac{k}{\sigma(\delta \mathrm{t})^{1-H}}\right)$. Finally, we get

$$
\frac{\partial V}{\partial t}+\frac{\sigma^{2}}{2} S^{2}(\delta \mathrm{t})^{2 H-1} \frac{\partial^{2} V}{\partial S^{2}}+\frac{\sigma^{2}}{2} L e(\mathrm{H}) \mathrm{S}^{2}\left|\frac{\partial^{2} V}{\partial S^{2}}\right|+r S \frac{\partial V}{\partial S}-r V=0 .
$$

For vanilla when $\frac{\partial^{2} V}{\partial S^{2}}<0$ the rate at which the loss occurs accelerates. That is why it is widely assumed that $\frac{\partial^{2} V}{\partial S^{2}}$ is always positive. Therefore, Equation (3.49) becomes

$$
\frac{\partial V}{\partial t}+\frac{\tilde{\sigma}^{2}}{2} S^{2} \frac{\partial^{2} V}{\partial S^{2}}+r s \frac{\partial V}{\partial S}-r V=0
$$

where

$$
\tilde{\sigma}^{2}=\sigma^{2}\left((\delta \mathrm{t})^{2 H-1}+L e(\mathrm{H})\right)
$$

Without loss of generality, we only consider the case of a European call option with terminal condition.

$\mathrm{V}(\mathrm{S}, \mathrm{T})=\max \{\mathrm{S}-\mathrm{E}, 0\}$ 
Citation: Nteumagn BF, Pindza E, Mare E (2017) Symmetry Analysis of Options Pricing with Transactions Costs Driven by Fractional Brownian Noises. J Appl Computat Math 6: 356. doi: 10.4172/2168-9679.1000356

We transform the problem into an initial value problem with the change of variables $\tau=\mathrm{T}-\mathrm{t}$. This leads to the PDE.

$$
\frac{\partial V}{\partial t}-\frac{\tilde{\sigma}^{2}}{2} S^{2} \frac{\partial^{2} V}{\partial S^{2}}-r S \frac{\partial V}{\partial S}+r V=0
$$

Subject to the initial conditions $\mathrm{V}(\mathrm{S}, 0)=\max \{\mathrm{S}-\mathrm{E}, 0\}$. The option pricing with transaction cost has a same form as the classical BlackScholes PDE, except for the transformation $\tau=\mathrm{T}-\mathrm{t}$ and the volatility parameter which is now given by eqn. (3.51). Proportional transaction costs do not affect the linearity of the equation when a vanilla option is considered. For the purpose of this work, we will focus on vanilla option pricing. The volatility structure of the $\mathrm{fBm}$ model depends on three important parameters: When we vary $\delta$, the optimal hedging interval, we are able to define the minimal volatility for this model as well as the equivalent time interval. The volatility structure is given in equation (3.51) above and Figure 1 plots the impact of changes in the Hurst parameter $\mathrm{H}$ on the volatility of the underlying stock.

$$
\frac{\partial \tilde{\sigma}}{\partial H}=\frac{\sigma\left(2 \delta t^{2 H-1}+\frac{k}{2} \sqrt{\frac{2}{\pi} \delta t^{H-1}}\right)}{2 \sqrt{\delta t^{2 H-1}+\frac{k}{2} \sqrt{\frac{2}{\pi}} \delta t^{H-1}}} \operatorname{In}(\delta t)
$$

Setting this to zero and solving for $\mathrm{H}$ leads to meaningless results in view of our model. However if we solve this in terms of $\delta \mathrm{t}$, we obtain

$$
\tilde{\sigma}_{\min }=\sigma \sqrt{2}\left(\frac{2}{\pi}\right)^{\frac{2 H-1}{4 H}}\left(\frac{k}{\sigma}\right)^{\frac{2 H-1}{2 H}}
$$

When $\delta t=\left(\frac{2}{\pi}\right)^{\frac{1}{2 H}}\left(\frac{k}{\sigma}\right)^{\frac{1}{H}}$. The profile of the minimal volatility is shown in Figure 2.

The complete volatility structure is shown in Figure 3. Notice that if $\mathrm{k}=0$ and $\mathrm{H}=0.5$, we have $\tilde{\sigma}=\sigma$.

Derivation of the geometric average Asian options pricing model under fractional Brownian motion

In addition to the assumptions of the European option, Asian

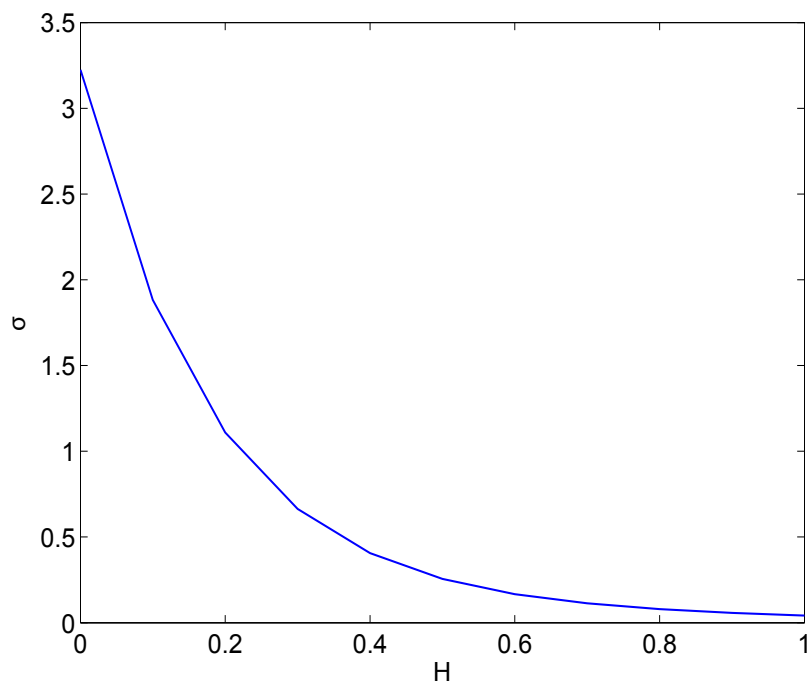

Figure 1: Volatility profile for $\tilde{\sigma}$ with $\mathrm{k}=0.002, \mathrm{H} \in[0,1] ; \mathrm{t}=\frac{1}{250}$.

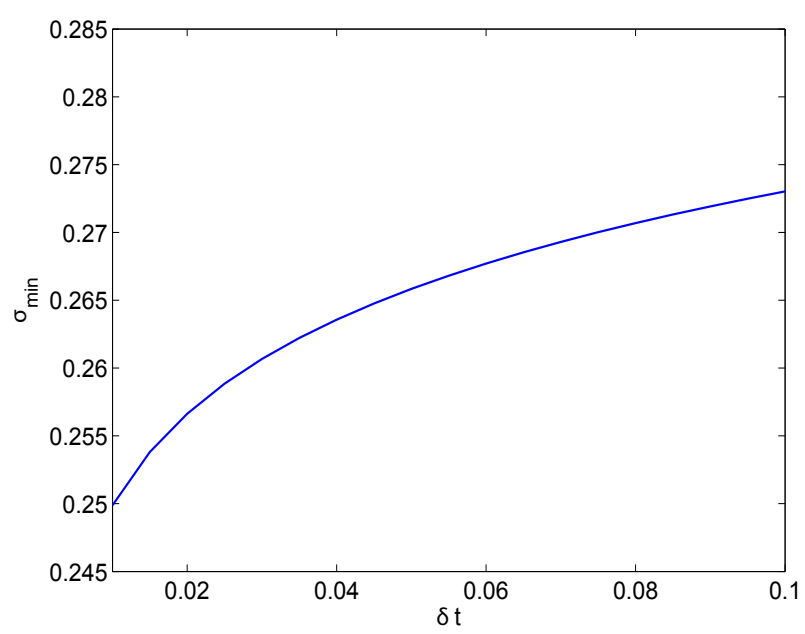

Figure 2: Minimal volatility profile for $\tilde{\sigma}$ with $\mathrm{H}=0.75$; $\mathrm{k}=0.002$; and $\delta \mathrm{t} \epsilon$ $\left[\frac{1}{250}, \frac{1}{12}\right]$.

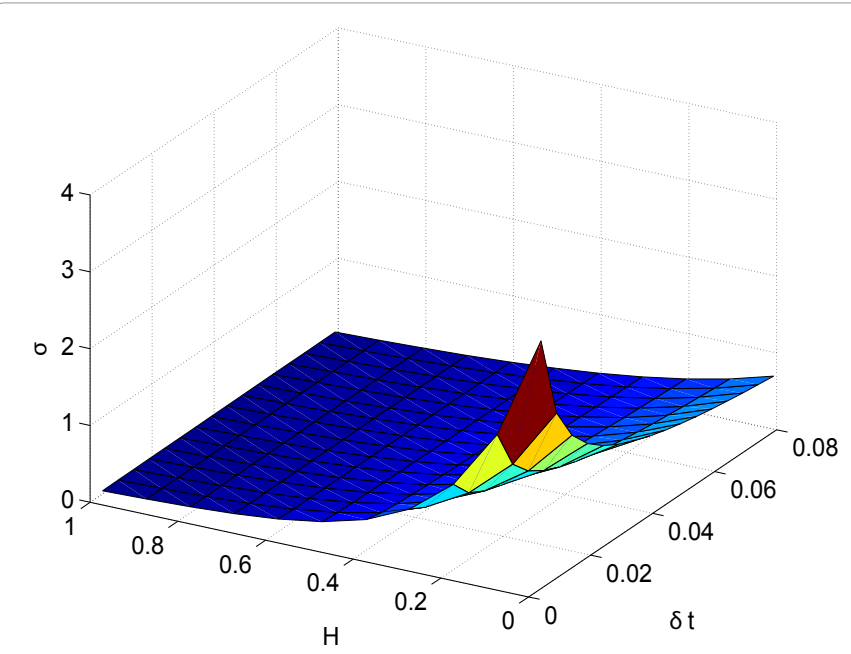

Figure 3: Volatility profile for $\tilde{\sigma}$ with $H \in[0,1]$; $k=0.002$; and $\delta t \in[1 / 250,1 / 12]$.

options posit that the stock price $\mathrm{x}(\mathrm{t})$ depends on historical prices. However the steps of the derivation of the equation are similar to those of the European option differential equation provided in the above section. We omit the details of the derivation for this reason.

The model in this context is given by

$$
\frac{\partial V}{\partial t}+\frac{1}{2} \tilde{\sigma}^{2} S^{2} \frac{\partial^{2} V}{\partial S^{2}}+r s \frac{\partial V}{\partial S}+\frac{\partial V}{\partial J} \frac{J \operatorname{In}(\mathrm{S} / \mathrm{J})}{t}-r V=0
$$

Where this time the volatility is time-dependent and is given by

$$
\begin{aligned}
& \tilde{\sigma}^{2}=2 \sigma^{2}\left(H t^{2 H-1}-L e(\mathrm{H}) \operatorname{sign}\left(\frac{\partial^{2} V}{\partial S^{2}}\right)\right) \\
& L e(\mathrm{H})=\frac{k}{\sigma}(\delta t)^{2} \sqrt{\frac{2}{\pi}} \text { the fractional Leland number, while }
\end{aligned}
$$

$\mathrm{t}$ and $\mathrm{S}$ remain the time and price of the stock respectively. 
Citation: Nteumagn BF, Pindza E, Mare E (2017) Symmetry Analysis of Options Pricing with Transactions Costs Driven by Fractional Brownian Noises. J Appl Computat Math 6: 356. doi: 10.4172/2168-9679.1000356

Page 6 of 8

$\mathrm{J}(\mathrm{t})=\frac{1}{t-t_{0}} \int_{t_{0}}^{t} \operatorname{In}(\mathrm{x}(\tau)) d \tau$ here denotes the geometric average of the stock price over the time interval $\left[\mathrm{t}_{0}, \mathrm{t}\right], \mathrm{V}=\mathrm{V}(\mathrm{S}, \mathrm{t}, \mathrm{J})$ and of course, the Hurst parameter $\mathrm{H}$. We can now proceed to do the symmetry analysis of these models. We start with the European option.

\section{Reduction of the Option Pricing Differential Equation and Solution}

We dedicate this section to solving the equations for pricing European and Asian options in eqn. (3.53) and (3.56). We first consider European option case, and later, the Asian options under fractional Brownian motion.

\section{European call option under fractional Brownian motion}

The first step is to transform Equation (3.53) into a heat like equation using the Lie symmetry analysis. Thanks to the computer algebra package Symteric $[12,13]$ one does not need to calculate the symmetries by hand, but a line of code success to verify that the symmetries of the PDE in eqn. (3.53) are as in ref. [10].

Equation (3.53) is reduced to the heat equation by applying the transformations

$$
\tau=\frac{t}{4 \bar{\sigma}^{2}}, y=\frac{\operatorname{In}(\mathrm{S} / \mathrm{E})}{2 \bar{\sigma}^{2}}, \mathrm{u}(\mathrm{y}, \tau)=\mathrm{e}^{\alpha \tau+\beta y} V(\mathrm{~S}, \mathrm{t})
$$

where $\alpha=\frac{\left(2 \mathrm{r}-\bar{\sigma}^{2}\right)^{2}}{2}$, and $\beta=-2 r+\bar{\sigma}^{2}$. In fact, the transformations of variables above give

$$
\begin{aligned}
& \frac{\partial u}{\partial S}=\frac{t}{2 \bar{\sigma}^{2}} \mathrm{e}^{\alpha \tau+\beta y}\left(\mathrm{u}_{y}(\mathrm{y}+\tau)+\beta \mathrm{u}(\mathrm{y}, \tau)\right) \\
& \frac{\partial u}{\partial t}=\frac{t}{8 \bar{\sigma}^{2}} \mathrm{e}^{\alpha \tau+\beta y}\left[\left(2 r+\tilde{\sigma}^{2}\right) \mathrm{u}(\mathrm{y}+\tau)+2 \bar{\sigma}^{2} u_{\tau}(\mathrm{y}, \tau)\right] \\
& \frac{\partial^{2} u}{\partial S^{2}}=\frac{t}{4 \bar{\sigma}^{4}} \mathrm{e}^{\alpha \tau+\beta y} \mathrm{~S}^{-\frac{r}{\bar{\sigma}^{2}-\frac{3}{2}}}\left[\left(4 r^{2}+\tilde{\sigma}^{4}\right) \mathrm{u}(\mathrm{y}, \tau)-4 \bar{\sigma}^{2} u_{y}(\mathrm{y}, \tau)+\tilde{\sigma}^{4} u_{y y}(\mathrm{y}, \tau)\right]
\end{aligned}
$$

Substituting these variables and their derivatives into Equation (5.53) yields the one dimensional heat equation

$$
\mathrm{u}_{r}=u_{y y}
$$

together with the initial condition

$u(\mathrm{y}, 0)=\mathrm{g}(\mathrm{y})=\mathrm{e}^{\alpha y} \max \left\{\mathrm{e}^{2 y}-\mathrm{E}, 0\right\}$

with solution

$$
\mathrm{u}(\mathrm{y}, \tau)=e^{(\alpha+2) \mathrm{y}+\frac{(\alpha+2)}{2} r \varnothing}\left(\frac{y+(\alpha+2) \tau}{\sqrt{\tau}}\right)-E e^{\alpha y+\frac{\alpha^{2}}{2}} \varnothing\left(\frac{y+\alpha \tau}{\sqrt{\tau}}\right)
$$

Defining new variables $d_{1}$ and $d_{2}$, then converting the variables back into the variables in our model, we have

$$
d_{1}=\frac{\operatorname{In}(\mathrm{S} / \mathrm{E})+\left(r+\frac{1}{2} \bar{\sigma}^{2}\right)(\mathrm{T}-\mathrm{t})}{\bar{\sigma} \sqrt{(\mathrm{T}-\mathrm{t})}} d_{2}=d_{1}-\bar{\sigma} \sqrt{(\mathrm{T}-\mathrm{t})}
$$

The solution of the PDE is obtained by backward substitution from $\mathrm{w}(\mathrm{y}, \tau)$ to $\mathrm{V}(\mathrm{S}, \mathrm{y})$. We get

$$
\mathrm{V}(\mathrm{S}, \mathrm{t})=\mathrm{SN}\left(\mathrm{d}_{1}\right)-\mathrm{Ee}^{\mathrm{r}(\mathrm{t}-\mathrm{T})} \mathrm{N}\left(\mathrm{d}_{2}\right),
$$

where $\mathrm{N}$ is the standard normal distribution probability mass function, which satisfies our equation and the terminal condition. Note that when $\mathrm{H}=0.5$ and $\mathrm{k}=0.002$, i.e., $0.2 \%$ transaction cost, we obtain the solution to the classical Black-Scholes equation. We vary the Hurst parameter and plot the solution in Figure 4.

We now consider the Asian option pricing problem in the same regime.

\section{Lie symmetry classification of the geometric Asian option pricing model under fractional Brownian motion}

In this subsection, we apply the theory described above to the Asian option pricing problem in $\mathrm{fBm}$. We consider the contextual model derived above in eqn. (3.56) and rewrite it for readability purposes as,

$$
r S V S-r V+\frac{1}{2} \bar{\sigma}^{2} S^{2} V s S+V_{i}+\frac{\operatorname{JIn}\left(\frac{S}{J}\right)}{t} V_{J}=0
$$

subject to the pay on

$\mathrm{V}(\mathrm{S}, \mathrm{T}, \mathrm{J})=\max \{\mathrm{J}-\mathrm{K}, 0\}$

where

$$
\bar{\sigma}^{2}=2 \bar{\sigma}^{2}\left(H t^{2 H-1}-\sqrt{\frac{2}{\pi} \frac{k}{\sigma}}(\delta \mathrm{t})^{H-1}\right)
$$

$\mathrm{V}=\mathrm{V}(\mathrm{S}, \mathrm{t}, \mathrm{J}), J=e^{(1 / \mathrm{t}) \int_{0}^{t} \operatorname{InS}(\mathrm{r}) \mathrm{dr}}$ is the geometric average of the price of the underlying $S$ since inception $\tau=0$ till time $\tau=t, K$ is the strike, $\mathrm{r}$ is the risk-free rate, which in most emerging markets is nonzero. $\sigma$ is a constant volatility. The minimum tradable interval $(\delta t)$ is very important for the practical aspect of our model. Note that if this is zero, it means that we are hedging in continuous time. It has, however, been shown (see for example [14]) that in continuous time we face the problem of arbitrage. In order to avoid the problem of arbitrage, we only consider the case where $(\delta \mathrm{t}) \neq 0$. The same condition applies to $\mathrm{k}$, the transaction cost. This is a proportion of turnover that would be charged for replicating the portfolio. If this is allowed to be $\mathrm{k}=1$, we would have more symmetries. However, it is clearly an unrealistic scenario. If the turnover on a transaction is equal to the cost of that transaction, it would never be worth entering such transactions. Despite the above, we find that in eqn. (4.65) in its full glory does not lead to any results. However, we distinguish the below cases:

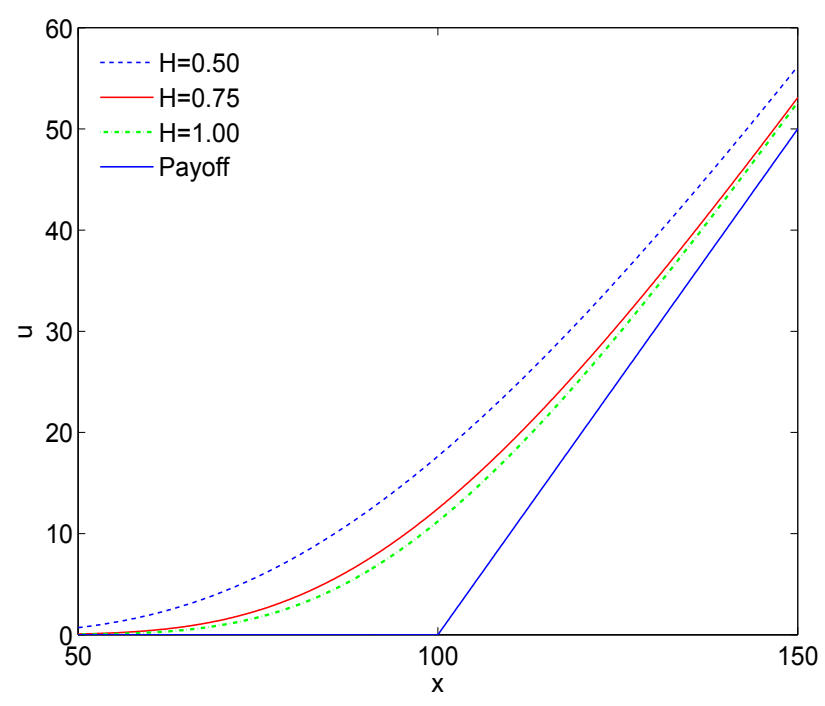

Figure 4: Volatility profile for $\tilde{\sigma}$ with $\mathrm{H} \in\{0.25,0.5,1\}$; $\mathrm{r}=0.02$; and $\mathrm{T}=5, \mathrm{k}=0.002$. 
Case $k=-\sqrt{\frac{\pi}{2}}\left(1-H t^{2 H-1}\right)(\delta \mathrm{t})^{1-H_{\sigma}}$

We have for this case, $\tilde{\sigma}=2 \sigma$, which gives the simplified model

$$
r S V s-r V+\sigma^{2} S^{2} V s s+V_{t}+\frac{J \log \left(\frac{S}{J}\right)}{t} V_{J}=0
$$

which admits the symmetries Lie algebra generators

$$
\begin{aligned}
& X_{1}=\partial_{t}-\frac{J}{t} \operatorname{In} J \partial_{j} \\
& X_{2}=s \partial_{s}+J \partial_{j} \\
& X_{3}=V \partial_{V}
\end{aligned}
$$

in addition to the infinite dimensional symmetry $\mathrm{f}(\mathrm{t}, \mathrm{S}, \mathrm{J}) \partial \mathrm{V}$, where

$$
r S f s-r f+\sigma^{2} S^{2} f s s+f_{t}+\frac{J \log \left(\frac{S}{J}\right)}{t} f_{J}=0
$$

Applying the symmetry $\mathrm{X}_{2}$ to perform the reduction, we have the quasi-linear PDE

$$
\frac{d S}{S}=\frac{d J}{J}=\frac{d t}{0}
$$

Which gives us the transformation variables

$\mathrm{y}(\mathrm{S}, \mathrm{J})=\mathrm{In} \mathrm{S}-\mathrm{In} \mathrm{J}, \quad \mathrm{V}(\mathrm{t}, \mathrm{S}, \mathrm{J})=\mathrm{u}(\mathrm{t}, \mathrm{y})$

Where u satisfies the $(1+1)$ PDE

$$
u_{t}+\left(\mathrm{r}+\sigma^{2}-\frac{y}{t}\right) \mathrm{u}_{y}+\sigma^{2} u_{y y}-r u=0
$$

The symmetry Lie algebra of eqn. 4.71 is spanned by the following operators:

$$
\begin{aligned}
X_{1}= & u d_{u} \\
X_{2}= & \frac{1}{t} \partial_{y} \\
X_{3}= & 4 t \partial_{t}+4 r t u \partial_{u}+\left(\mathrm{rt}+\sigma^{2} t+2 y\right) \partial_{y} \\
X_{4}= & \left(-3 t^{2}-\frac{3 r t^{2}}{\sigma^{2}}+\frac{6 t y}{\sigma^{2}}\right) u \partial_{u}+\left(4 \mathrm{t}^{2}\right) \partial_{y} \\
X_{5}= & \frac{1}{t^{2}} \partial_{t}+\left(\frac{r}{t^{2}}+\frac{\sigma^{2}}{t^{2}}-\frac{y}{t^{3}}\right) \partial_{y}+\frac{r u}{t^{2}} \partial_{u} \\
X_{6}= & \left(16 \mathrm{t}^{4}\right) \partial_{t}+\left(-8 \mathrm{rt}^{4}-8 \sigma^{2} t^{4}+32 \mathrm{t}^{3} \mathrm{y}\right) \partial_{y} \\
& +\left(-24 t^{3}+34 r t^{4}+\frac{9 r^{2} t^{4}}{\sigma^{2}}+9 \sigma^{2} t^{4}-36 t^{3} y-\frac{36 r t^{3}}{\sigma^{2}}+\frac{36 t^{2} y^{2}}{\sigma^{2}}\right) u \partial_{u} \\
r_{\infty}= & \int(\mathrm{t}, \mathrm{y}) \partial_{u}
\end{aligned}
$$

Where $\mathrm{f}(\mathrm{t}, \mathrm{y})$ satisfies the PDE

$$
f_{t}+\left(r+\sigma^{2}-\frac{y}{t}\right) f_{y}+\sigma^{2} f_{y y}-r f=0
$$

Reduction of (4.71) is therefore possible by means of the transformation $\tau=-4 \sigma 2 / \mathrm{t} 5, \mathrm{z}=\mathrm{y} / \mathrm{t} 2-\mathrm{rt}-\sigma 2 \mathrm{t}$,

$\tau=-4 \sigma^{2} / t^{5}, \mathrm{z}=\mathrm{y} / \mathrm{t}^{2}-r t-\sigma^{2} t, u(\mathrm{t}, \mathrm{y})=w(\tau, \mathrm{z}) \mathrm{e}^{-r t}$

to the heat equation

$w_{r}+w_{z z}=0$,

and the solution of the latter is $w(\tau, \mathrm{z})=\mathrm{e}^{z+r} \aleph\left(\mathrm{c}_{1}\right)-\mathrm{E} \aleph\left(\mathrm{c}_{2}\right)$

with $c_{1}=\frac{z+2 \tau \operatorname{In}(\mathrm{E})}{\sqrt{2 \tau}}$ and $c_{2}=\frac{z-\operatorname{In}(\mathrm{E})}{\sqrt{2 \tau}}$.

Substituting back the transformation concludes this case.

Case $\mathrm{k}=0,(\delta \mathrm{t}) \neq 0$

In the case of no transactions cost, we find no symmetry that enables us to perform reduction of the PDE.

Case $k=\sqrt{\frac{\pi}{2}} \frac{H \sigma}{(\delta \mathrm{t})^{H-1}}, \sigma=1 / \sqrt{2 H}, r=0$

This condition yields

$\frac{J(\operatorname{InS}-\operatorname{In} \mathrm{J})}{t} V_{t}+\frac{1}{2}\left(-1+\mathrm{t}^{-1+2 H}\right) \mathrm{S}^{2} V_{S S}+V_{t}=0$

with basis for the symmetries Lie algebra given by

$X_{1}=-\frac{S}{t} \partial s+\frac{J}{t} \partial_{j}, X_{2}=V \partial_{V}$

as well as the infinite dimensional symmetry. The reduction process using $\mathrm{X}_{1}$ yields the transformation

$\xi=t(\operatorname{InJ}-\operatorname{In} S), \mathrm{t}=\mathrm{t}, \mathrm{V}(\mathrm{t}, \mathrm{S}, \mathrm{J})=\mathrm{V}(\mathrm{t}, \xi)$

We substitute the above into (4.77) to obtain the $1+1 \mathrm{PDE}$

$V_{t}+\frac{1}{2}\left(\mathrm{t}^{2 H}-\mathrm{t}\right) \mathrm{V}_{\xi}+\frac{1}{2}\left(\mathrm{t}^{2 H+1}-t^{2}\right) \mathrm{V}_{\xi \xi}=0$

Which admits the following basis for the symmetry algebra.

$$
\begin{aligned}
& X_{1}=\frac{1}{-t^{2}+t^{2 H}} \partial_{t} \\
& X_{2}=\partial_{\xi} \\
& X_{3}=V \partial_{V} \\
& X_{4}=-\frac{\left((2+2 H) \mathrm{t}^{2}-3 \mathrm{t}^{2 H+1}\right)}{(6+6 \mathrm{H})\left(-\mathrm{t}+\mathrm{t}^{2 H}\right)} \partial_{V}-\left(\xi+\frac{t^{2 H+1}}{4 H+2}-\frac{t^{2}}{4}\right) \partial_{\xi} \\
& X_{5}=\left(\xi+\frac{1}{2}\left(\frac{t^{2 H+1}}{2 H+1}-\frac{t^{2}}{2}\right)\right) V \partial_{V}-\frac{(2+2 \mathrm{H}) \mathrm{t}^{2}-3 t^{2 H+1}}{(6+6 H)\left(-\mathrm{t}+\mathrm{t}^{2 H}\right)} \partial_{\xi} \\
& X_{6}=\frac{\left((2+2 \mathrm{H}) \mathrm{t}^{4}-3 \mathrm{t}^{2 H+3}\right)^{2}}{(1+H)^{2}\left(-\mathrm{t}+\mathrm{t}^{2 H}\right)} \partial_{t}+\left(\frac{t^{3}}{3}-\frac{t^{2+2 H}}{2+2 H}+\left(\frac{t^{2}}{4}-\frac{t^{1+2 H}}{2+4 H}\right) \xi\right) V \partial_{V} \\
& \Gamma_{\infty}=f(\mathrm{t}, \xi) \partial_{u}
\end{aligned}
$$

where $\mathrm{f}$ satisfies $f_{t}+\frac{1}{2}\left(\mathrm{t}^{2 H}-\mathrm{t}\right) f_{\xi}+\frac{1}{2}\left(\mathrm{t}^{2 H+1}-\mathrm{t}^{2}\right) f_{\xi \xi} \mathrm{ft}+1 \quad 2(\mathrm{t} 2 \mathrm{H}-\mathrm{t}) \mathrm{f} \xi+1$ $2(\mathrm{t} 2 \mathrm{H}+1-\mathrm{t} 2) \mathrm{f} \xi \xi=0$. It is worth noting that the generators of symmetries above were obtained by transforming those of the heat equation. Using the normal algorithm for generating the symmetries in Section 2, we obtain determining equations, of which the closed form solutions are difficult to construct equation (4.80) can be transformed to the heat equation by the use of the change of independent variables

$$
\tau=\frac{1}{2}\left(\frac{t^{3}}{3}-\frac{t^{2 H+2}}{2 H+2}\right), y=\xi+\frac{1}{2}\left(\frac{t^{2 H+1}}{2 H+1}-\frac{t^{2}}{2}\right)
$$

and the solution is as in case in eqn. 4.2.1. Applying the solution process in as per Case 4.2.1 concludes this section.

\section{Concluding Remarks}

European and geometric average Asian options were studied in this work. We successfully constructed the symmetries of the European 
option pricing model and provided the mathematical tools for mapping this model to a known PDE. Despite the transaction cost and the source of randomness, the model keeps similar algebraic structure to the classical Black-Scholes equation. In other words the model with transaction cost, when considering a problem of a vanilla option, keeps its classification. Some suggestions were given to relax the assumption on the sign of $\Gamma$, however, the problem becomes ill-posed if $\Gamma<0$.

For geometric average Asian options, we have provided a classification of the PDE and closed form solutions were provided whenever it was possible. We notice that though there has been some progress in technology, it is still relevant and important to keep the rigor of the algorithm. This is due to the fact that though some computer algebras are capable to handle generic systems, it is difficult to obtain symmetries of some PDEs. As an example, with the parametric time dependency of the PDE for the Asian option, we were unable to apply Sym to obtain the symmetries. We had to fall back on Ya Lie, in order to regain control of the solution process and in the end, we realized that not all the determining equations were solvable using the normal solution process in Mathematical. It is in this regard that we opted to exploit a well known use of symmetries to transform the symmetries of the heat equation.

Methods that have been used so far to obtain the solutions of the above problems are ad hoc in nature. We acknowledge that the application of Lie symmetries analysis provided here is systematic and can be automated. Applying equivalence transformations to the reduced model, we transformed these to the heat equation and provided a solution thereof. We have limit ourselves to European option pricing with constant volatility. We know, however, that in real market conditions, the volatility is not constant. Considering stochastic volatility in $\mathrm{fBm}$ would be an extension of this work. Additionally, it will be interesting to now calibrate these models and estimate $\mathrm{H}$ for a specific asset class in a given period of time. A question of interest may be: Could non-market conditions dictate the level of the Hurst parameter? In other words, what would have been the effect of the 2008 crisis to $\mathrm{H}$ for a specific product type? Can $\mathrm{H}$ capture a similar market reaction to another crisis in future? Answers to these question could be an interesting extension to the building of these models.

\section{Acknowledgements}

This work was partially financed by Brad Welch at Ridge Cape Capital. This financial assistance enabled BFN to present this work at AIMS in Orlando. BFN would like to express his sincere appreciations to Prof. C. Wafo Soh for his feedback and advice during the preparation of this manuscript.

\section{Declaration}

The authors declare that there is no conflict of interest regarding the publication of this paper.

\section{References}

1. Zhang Y, Pan D, Zhou SW, Han M (2014) Asian option pricing with transaction costs and dividend's under the fractional Brownian motion model. Journal of Applied Mathematics Hindawi Publishing Corporation, p: 8.

2. Bluman GW, Kumei S (1989) Symmetries and differential equations. Applied Mathematical Sciences Springer-Verlag 81: 229-237.

3. Sophocleous C, OHara JG, Leach PGL (2011) Symmetry analysis of a model of stochastic volatility with time-dependent parameters. Journal of Computational and Applied Mathematics 235: 4158-4164

4. Nualart D (2006) Fractional Brownian motion: Stochastic calculus and applications. Proceedings of the International Congress of Mathematicians 3: 1541-1560.

5. Hull JC (2012) Options, futures and other derivatives. Pearson, 8th edition.
6. Mandelbrot BB, Van Ness JW (1968) Fractional Brownian motion, fractional noises and applications. SIAM Review 10: 422-437.

7. Dai W, Heyde CC (1996) It's formula with respect to fractional Brownian motion and its application. Journal of Applied Mathematics and Stochastic Analysis 9: 439-448.

8. Hurst HE (1987) Long-term storage capacity in reservoirs, transactions of the American Society of Engineers116: 770-799.

9. Ibragimov NH, Kara AH, Mahomed FM (1997) Lie-Backlund" and Noether symmetries with applications. Nonlinear Dynamics 15: 115-136.

10. Ibragimov NH, Gazizov RK (1998) Lie symmetry analysis of differential equations in finance. Nonlinear Dynamics 17: 387-407.

11. Leland HE (1985) Option pricing and replication with transaction costs. Journa of Finance 40: 1283-1301.

12. Dimas S, Tsoubelis D (2004) SYM: A new symmetry-finding package for Mathematica. Proceedings of the 10th International Conference in Modern Group Analysis, p: 24-30.

13. Dimas S, Tsoubelis D (2006) A new Mathematica-based program for solving over determined systems of PDE. Proceedings of the 8th International Mathematica Symposium, p: 19-23.

14. Cheridito $P(2003)$ Arbitrage in fractional Brownian motion models. Finance and Stochastics 7: 533-553.

15. Wilmott P, Howison S, Dewynne J (1995) The Mathematics of financial derivatives. Cambridge University Press.

16. Mahomed FM (2008) Complete invariant characterization of scalar linear $(1+1)$ parabolic equations. Nonlinear Mathematical Physics 1: 112-123

17. Olver PJ (1986) Applications of Lie groups to differential equations. Graduate texts in Mathematics.

18. Ovsiannikov LV (1982) Group analysis of differential equations.Academic Press, p: 482

19. Nteumagne BF, Pindza E, Mare E (2014) Applying the barycentric Jacobi spectral method to price options with transaction costs in a fractional BlackScholes framework. Journal of Mathematical Finance 4: 35-46.

20. Ablamovitz A, Stagun N (1970) Handbook of Mathematical functions with formulas, graphs and Mathematical tables.

21. Houle ME, Kreigel HP, Kroger P, Schubert E, Zimek A (2001) Quality of similarity rankings in time series. Advances in spacial and temporal database 6849: 422.

22. Gazizov RK, Ibragimov NH (1998) Lie symmetry analysis of differential equations in finance. International Journal of Nonlinear Dynamics 17: 387-407.

23. Goard J (2000) New solutions to the bond-pricing equation via Lie's classical method. Mathematical and Computer Modelling 32: 299-313.

24. Pooe CA, Mahomed FM, Wafo Soh C (2004) Fundamental solutions for zerocoupon bond-pricing models. Nonlinear Dynamics 36: 69-76.

25. Paulian AD, Zaitsev VF (2002) Handbook of exact solutions for ordinary differential equations. Chapman \& HALL CRC

26. Wang XT (2010) Scaling and long-range dependence in option pricing I: Pricing European option with transaction costs under fractional Black-Scholes model Physics A 389: 438-444

27. Wang XT, Zhu EH, Tang MM, Yan HG (2010) Scaling and long-range dependence in option pricing II: Pricing European option with transaction costs under the mixed Brownian-fractional. Brownian model Physics A 389: 445-451.

28. Wang XT (2010) Scaling and long-range dependence in option pricing I: Pricing European option with transaction costs under the multifractional. Black-Scholes model Physics A 389: 789-796. 